[Technical Paper]

\title{
Identification of Through-Silicon Via (TSV) Failure Points Using the Time Domain Reflectometry (TDR) Method
}

\author{
Keiji Matsumoto*, Hiroaki Otsuka**, Osamu Horiuchi***, Young Gun Han***, Woon Choi**, \\ and Hajime Tomokage**,*** \\ *Department of Electrical and Electronic Engineering, Kitakyushu National College of Technology, 5-20-1 Shii, Kokuraminami, Kitakyushu, Fukuoka \\ 802-0985, Japan \\ **Department of Electronics Engineering and Computer Science, Fukuoka University, Fukuoka 814-018, Japan \\ ***Center of System Integration Platform Organization Standards, Fukuoka 819-1122, Japan
}

(Received May 13, 2013; accepted November 6, 2013)

\begin{abstract}
Time domain reflectometry (TDR) is applied to the failure analysis of through-silicon vias (TSVs). The reflection time of the TDR signal from test-element-group (TEG) chips with TSVs stacked on a silicon interposer is compared to the distance between the failure point and the contact pad derived from a TDR simulation value, using a model which has the same structure as the TEG chips. These results show good agreement between the simulated and the measured results.
\end{abstract}

Keywords: TSV, TDR, Failure Analysis, Time Derivative, Simulation

\section{Introduction}

In recent years, 3D integration technologies that have the capability to realize high-density packaging have become desirable because of the increasing demand for miniaturization and high performance. Although chip stacking technologies such as wire-bonding have been adopted in many applications, a new paradigm to overcome the limitations of interconnect performance is required for future advancements. In a 3D IC, TSV is the leading technology trend that might replace the traditional interconnection method. Because TSVs pass through the silicon substrate vertically, the increasing demands of electrical performance are satisfied. Recently, the electronic performance of TSVs at high frequencies has been studied, and many investigations related to this topic have been reported.[1-3] TSVs have become an important technology in advanced chips, packages, and systems. However, in the case of multi-stacked chips connected using metal bumps, the package might suffer from a poor connection due to thermal stress or initial implementation failure. Therefore, a new evaluation technique for interconnection failures and defects is required. Although X-ray observation and scanning acoustic microscopy are possible methods for detecting defects in TSVs, electrical characterization by a non-destructive method is preferable.
TDR is able to measure the characteristic impedance of a transmission line using a reflective wave and is widely used in high-speed signal design. This method is used extensively for the estimation of static electrical conductivity,[4] for the measurement of capacitance,[5] and for extracting an RLC circuit model from a distributed constant circuit based on a microstripline.[6] Moreover, TDR has been studied in many different fields for the evaluation of cracking in reinforced concrete structures, [7] the monitoring of liquids, [8] and so on. Kwon et al. reported on a nondestructive sensing method for interconnect failure mechanisms using TDR. [9] Solder cracking or separation of the solder pad from the circuit board caused the reflection coefficient of the TDR signal to gradually decrease. They proposed that interconnect failures with these characteristics can be more easily discovered using TDR than by traditional testing methods based on electrical resistance monitoring. However, their method cannot determine the exact failure location. In other research, Chen $e t$ $a l$. reported on the nondestructive analysis of interconnection failure points on a ball grid array package using TDR. [10] Although the failure locations that formed in the substrate were determined using this nondestructive method, they do not explain the detection accuracy in detail. Consequently, there are currently no reports of the use of TDR 
to detect the exact location of TSV failure points.

In this paper, TDR is applied to the failure analysis of TSVs. The reflection time of the TDR signal from the TEG chips with TSVs stacked on a silicon interposer is compared to the distance between the failure point and the contact pad using the simulated TDR value, where the simulation uses the same structure as the TEG chips.

\section{Principles of the TDR Measurement Method}

The TDR measurement method is shown in Fig. 1. Either a step voltage or a high-speed pulse is applied to the line in the substrate, and the reflected waveform indicates the difference of the characteristic impedance. When the termination is open as shown in Fig. 1 (a), the reflected voltage becomes twice that of the input voltage. In the case of a short [Fig. 1 (b)], the observed signal becomes zero because of the negative reflective waveform. The reflection time corresponds to the electrical length and is equal to the velocity of light in a vacuum divided by the square root of the effective relative permittivity. A Tektronix TDS8000 digital sampling oscilloscope, which is able to perform approximately $50-\mathrm{GHz}$ band frequency TDR measurements and provides the $15 \mathrm{ps}$ rise time for the step voltage, was used in the experiment.

\section{Measurement Sample}

Figure 2 (a) shows a top view of a fabricated sample. The sample is $14 \mathrm{~mm}$ wide $\times 14 \mathrm{~mm}$ long $\times 400 \mu \mathrm{m}$ thick. The same four structures were formed at intervals of 150 $\mu \mathrm{m}$ on the substrate for measurement reproducibility. These structures are numbered from \#1 to \#4. A schematic view of the structure used in this experiment is shown in Fig. 2 (b). The four thin chips are stacked on the silicon

\section{Open circuit termination (a)}

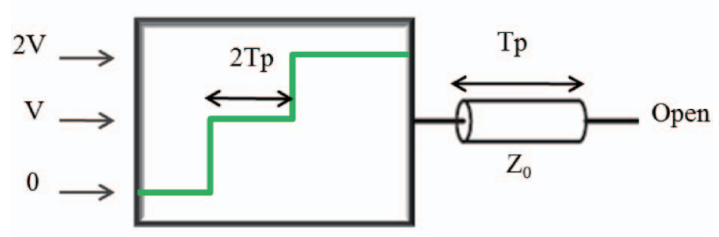

Short circuit termination (b)

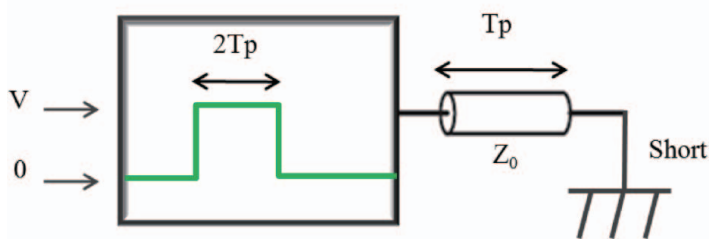

Fig. 1 Principle of the TDR measurement. interposer, and two TSVs are fabricated in each chip. The cross section and specifications of the interposer are shown in Fig. 3. The electrode pads for the measurement of the electrical characteristics formed in the ground-signal-ground (GSG) structure are located at intervals of 200 $\mu \mathrm{m}$ on the silicon interposer using $\mathrm{Cu}$. The diameters of the vias are $50 \mu \mathrm{m}$, and the insulating materials between the $\mathrm{Si}$ and $\mathrm{Cu}$ inside the vias consist of $0.4 \mu \mathrm{m}$ thick SiN and $2.6 \mu \mathrm{m}$ thick $\mathrm{SiO}_{2}$. The method of forming the $\mathrm{Cu}$ of the TSV is different from that of an internal wiring. The hole of the TSV is filled using Cu electrolytic plating after forming a seed film using sputtering. Afterwards, the surface is flattened by chemical mechanical planarization. For internal wiring, the wiring pattern is formed using a photolithography process after forming a seed film on an organic membrane. After that, the wiring line is processed by $\mathrm{Cu}$ electroplating, and the resist is stripped. The $\mathrm{Ni} / \mathrm{Au}$ plating is processed for surface passivation, and a PI coating is applied to both the top and the back surfaces to prevent oxidation. Figure 4 shows the cross section and specifications of the TEG chips stacked on a silicon interposer, called the middle chip and return chip, respectively. The middle and return chips are both $10 \mathrm{~mm}$ wide $\times 10 \mathrm{~mm}$ long $\times 50 \mu \mathrm{m}$ thick. The $\mathrm{Cu}$ post and solder are not included in this thickness. The insulating layers between
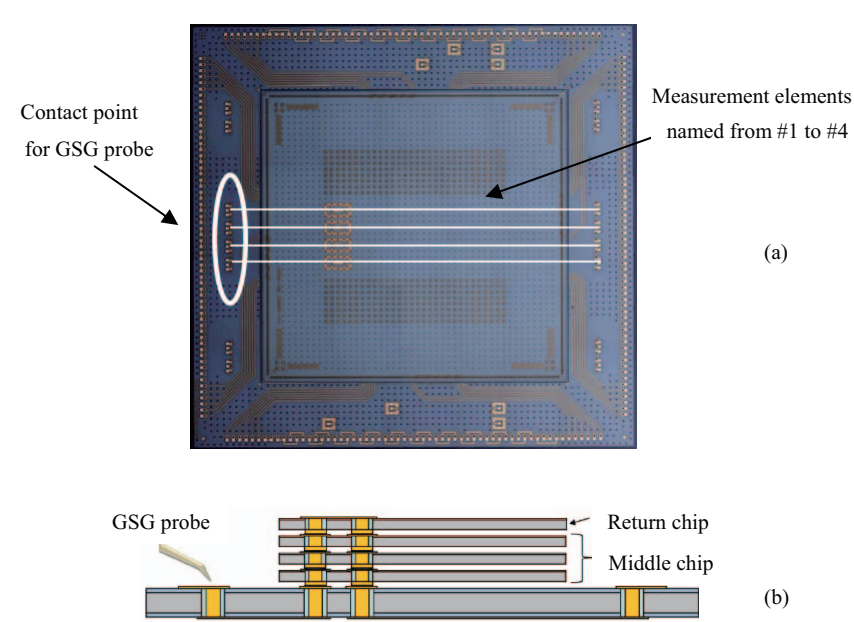

(b)

Fig. 2 Cross section of the measurement element TSV chip stacked on interposer.

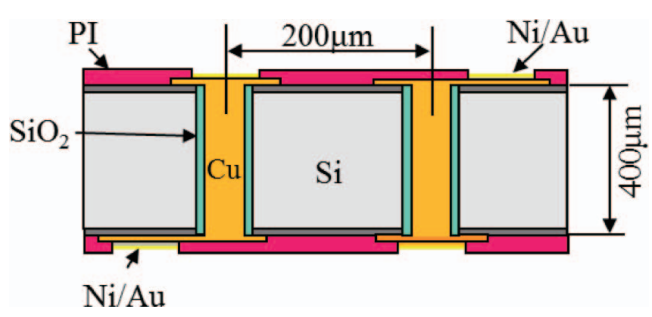

Fig. 3 Cross section of interposer. 

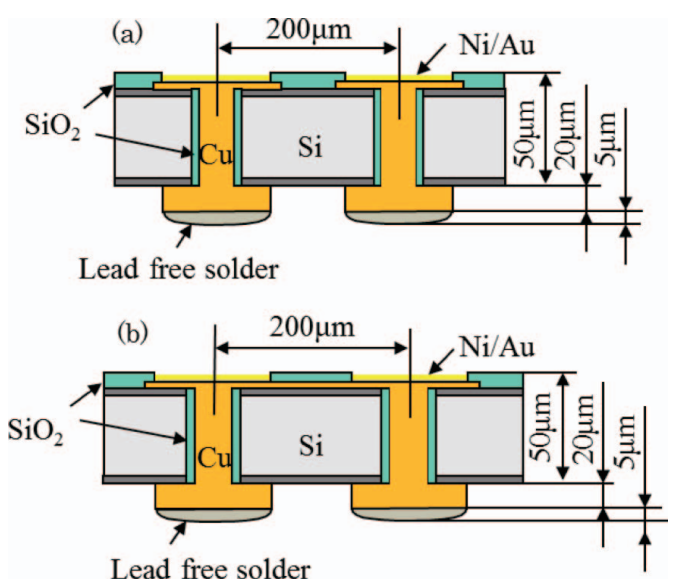

Fig. 4 Cross section of (a) a middle chip and (b) a return chip.

the $\mathrm{Cu}$ and $\mathrm{Si}$ are $0.4 \mu \mathrm{m}$ thick $\mathrm{SiN}$ and $2.6 \mu \mathrm{m}$ thick $\mathrm{SiO}_{2}$. The TSVs are $40 \mu \mathrm{m}$ in diameter. The post on the back is $\mathrm{Cu}$, and lead-free solder, composed of a combination of $\mathrm{Sn}$, $\mathrm{Ag}$, and $\mathrm{Cu}$, is used for making contact.

\section{Results and Discussion}

Figure 5 (a) shows the TDR signal of the contact probe itself including a coaxial cable. Because the contact probe is in an open state, the reflected waveform returns to the probe tip. To determine the reflection time, the derivative of the signal in Fig. 5 (a) with respect to time is shown in Fig. 5 (b). The peak position of $29.05 \pm 0.02$ ps was taken as the electrical length of the contact probe, including the coaxial cable.

The connection failure points, numbered from 1 to 7 , were formed in the sample substrate, as shown in Fig. 6. The distances between the GSG contact pad and each point were measured using cross-sectional observation after the TDR measurement. Figure 7 (a) shows the TDR signal of the sample for a failed connection at Point 7 , and Fig. 7 (b) shows the time derivative of the signal. An enlarged view of the signal near the peak point is shown in Fig. 8. The solid line was obtained by fitting the data with a Gaussian function. From this result, the electrical length up to the peak point was found to be 111.09 ps after subtracting the electrical length of the contact probe. In the four lines from \#1 to \#4, the standard deviation of the electrical length up to the peak point was $0.052 \mathrm{ps}$. The measurement results for each point are presented in Table 1. The error of the measurement results was less than $0.1 \mathrm{ps}$ for every point. Therefore, the measurements were confirmed to be repeatable. The reason for the value of Point 7 , \#3 in Table 1 is that a failure point could not be formed.

Next, a TDR simulation was carried out to confirm the

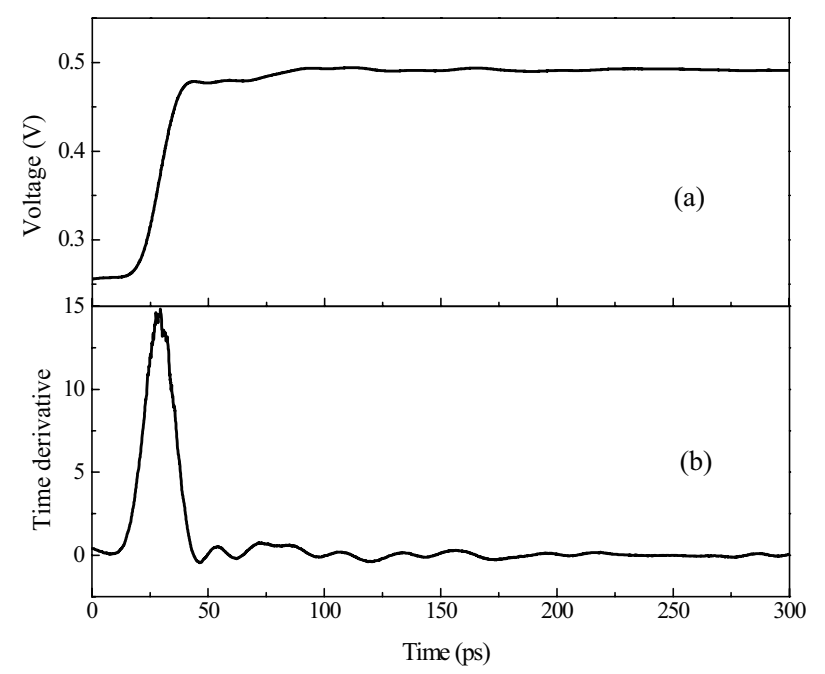

Fig. 5 TDR waveform (a) and differentiated waveform (b) in an open state.

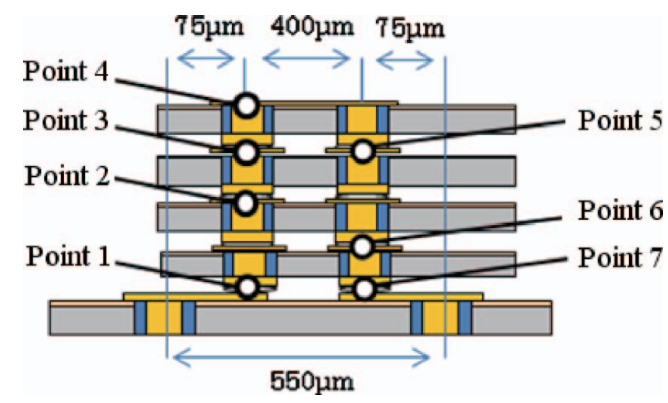

Fig. 6 Location of the failure points.

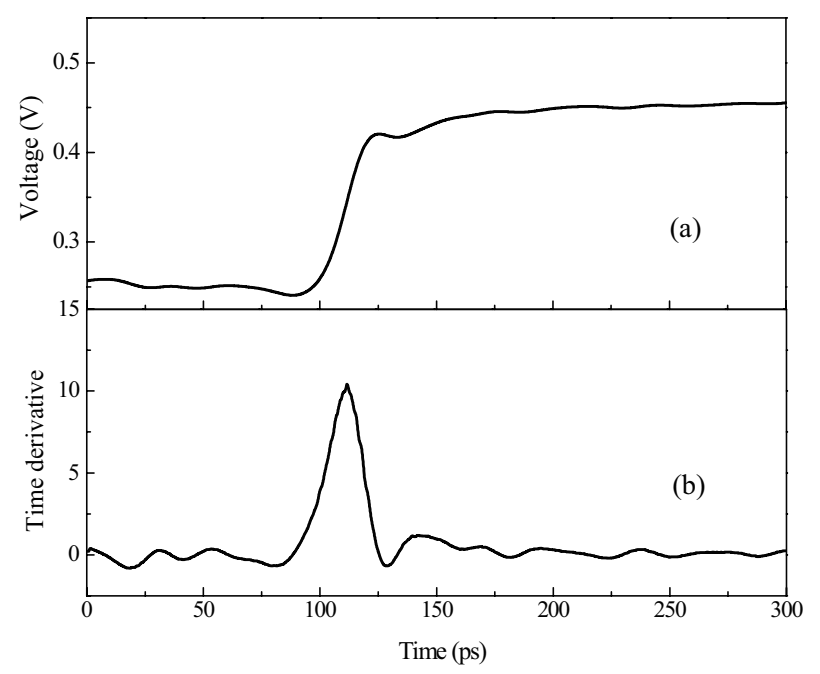

Fig. 7 TDR waveform (a) and differentiated waveform (b) at point 7 .

precise failure location. Figure 9 shows the simulation model, which has the same structure as the fabricated sample. Connection failure points were also formed in the simulation model at the same locations. The waveforms obtained using the TDR simulation are shown in Fig. 10. The time derivatives of these waveforms were obtained in the same way as the measurements, and their results are 


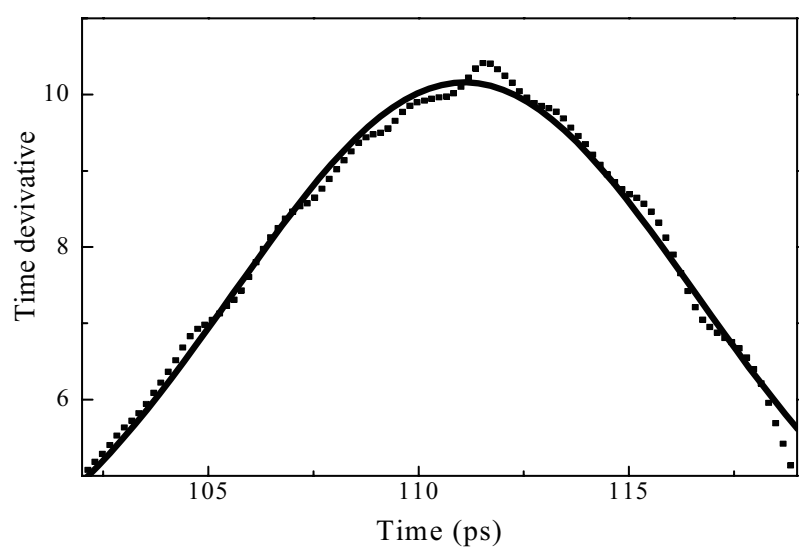

Fig. 8 Differentiated waveform at point 7.

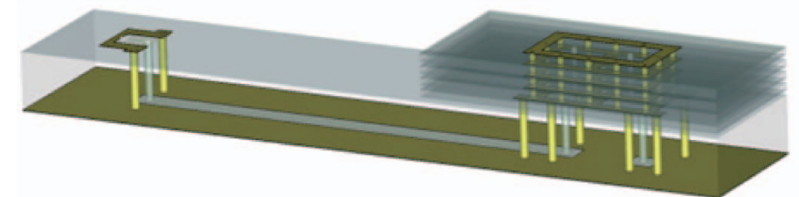

Fig. 9 Simulation model which has same structure to the TEG chip.

Table 1 Measured electrical length of the each failure point.

\begin{tabular}{l|l|l|l|l|l|c}
\hline & $\# 1(\mathrm{ps})$ & $\# 2(\mathrm{ps})$ & $\# 3(\mathrm{ps})$ & $\# 4(\mathrm{ps})$ & $\begin{array}{c}\text { Average } \\
(\mathrm{ps})\end{array}$ & $\begin{array}{c}\text { Standard } \\
\text { deviation }\end{array}$ \\
\hline Point 1 & 32.83 & 32.97 & 32.94 & 32.94 & 32.92 & 0.062 \\
\hline Point 2 & 34.1 & 34.09 & 34.08 & 34.19 & 34.11 & 0.051 \\
\hline Point 3 & 34.7 & 34.72 & 34.71 & 34.77 & 34.72 & 0.031 \\
\hline Point 4 & 35.12 & 35.13 & 35.06 & 35.24 & 35.14 & 0.075 \\
\hline Point 5 & 37.88 & 37.75 & 37.93 & 37.98 & 37.89 & 0.099 \\
\hline Point 6 & 39.98 & 40.01 & 40.04 & 39.96 & 40 & 0.035 \\
\hline Point 7 & 41.02 & 41.11 & 109.08 & 41.02 & 41.05 & 0.052 \\
\hline
\end{tabular}

shown in Fig. 11. The simulation model is constructed and analyzed based on the fabricated sample. Therefore, the complication is attributed to the slight impedance mismatch existing in the simulation model.

Figure 12 shows the simulated and the measured results. The vertical axis is the electrical length, and the horizontal axis is the distance from the contact pad to each failure point. The straight solid line was calculated from the measurement results using the least-squares method. The straight dotted line was calculated in the same way. These simulated results show good agreement with the measured results.

To identify a failure point, the relationship between the electrical length and the distance must be simulated in advance. For this purpose, all poor connection points arising from a variety of causes are assumed in the simulation model, and a TDR simulation is performed for every point. After the simulation, the relation between the electrical length and the distance shown in Fig. 12 is determined by plotting the results. If incorrect data regarding existing failure points in the substrate are obtained from the TDR measurement, it is possible to find the problematic location by comparing this data to the simulated relation

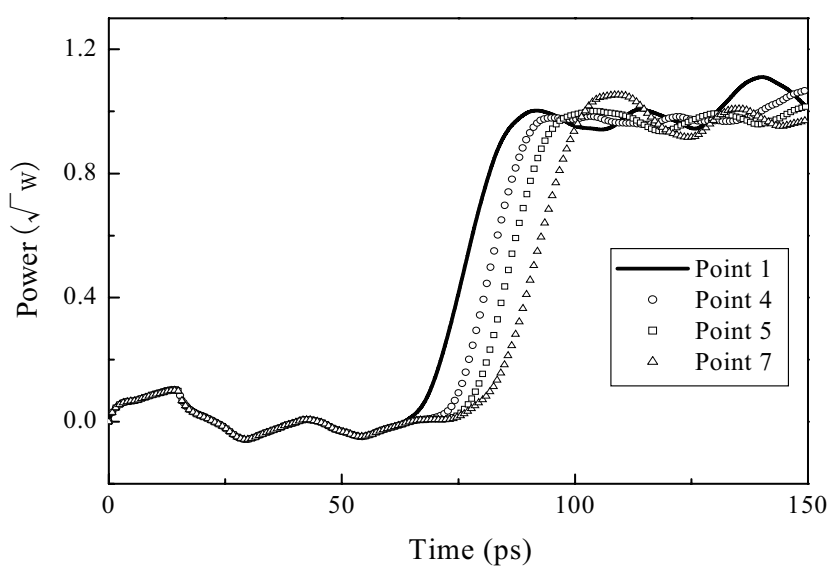

Fig. 10 TDR Simulation waveform.

graph. Although the diameters of TSVs have been decreasing recently, it is possible to apply this method as long as the impedances in the transmission lines including the TSV are matched. Therefore, we suggest that the failure points of TSVs can be identified using TDR signals.

\section{Conclusion}

The TDR signals of TEG chips stacked with a TSV structure on a silicon interposer were measured. The relation between the location of a connection failure point and the 


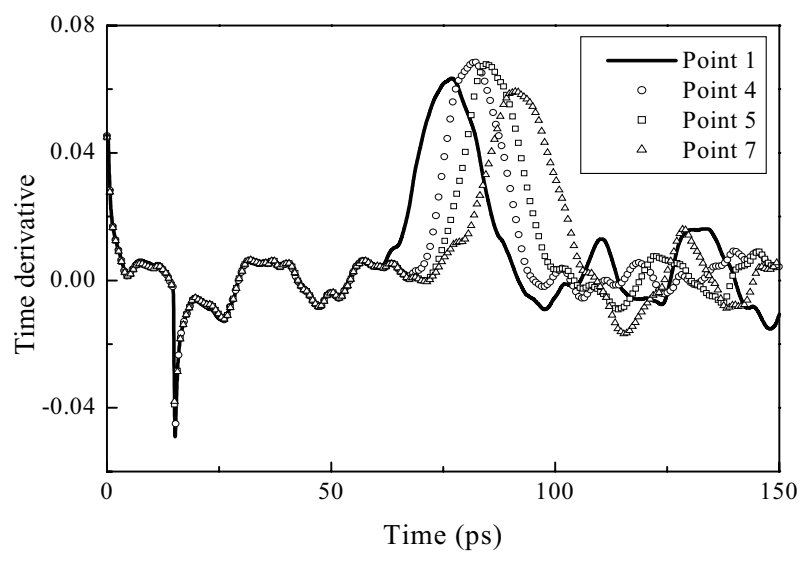

Fig. 11 Differentiated waveform of the TDR simulation.

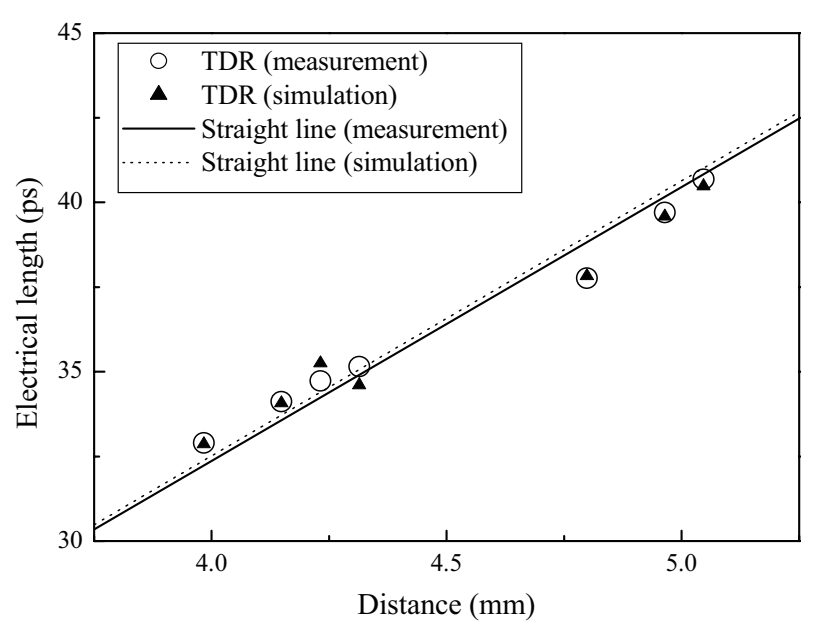

Fig. 12 Relationship between the electrical length and distance.

peak point obtained from the time derivative of a reflected wave was investigated. The error in the timing of the TDR measurement was less than $0.1 \mathrm{ps}$ for every point. A TDR simulation was carried out using a model with the same structure as the measured TEG chips, and the results showed good agreement. To locate a failure point, the relationship between the electrical length and the distance must be simulated in advance. It is possible to find a problematic location by comparing the TDR signal to the simulated results. Therefore, it is suggested that the failure points in TSVs can be identified using the TDR signal.

\section{References}

[1] S. W. Ho, S. W. Yoon, Q. Zhou, K. Pasad, V. Kripesh, and J. H. Lau, "High RF Performance TSV Silicon Carrier for High Frequency Application,” IEEE Electronic Components and Technology Conference, pp. 1946-1952, 2008.

[2] J. Kim, J. S. Pak, J. Cho, E. Song, J. Cho, H. Kim, T.
Song, J. Lee, H. Lee, K. Park, S. Yang, M.-S. Suh, K.-Y. Byun, and J. Kim, "High-Frequency Scalable Electrical Model and Analysis of a Through Silicon Via (TSV)," IEEE Transactions on Components, Packaging, and Manufacturing Technology, Vol. 1, No. 2, pp. 181-195, Feb. 2011.

[3] Z. Xu, Student Member, IEEE, and J.-Q. Lu, "HighSpeed Design and Broadband Modeling of ThroughStrata-Vias (TSVs) in 3D Integration,” IEEE Transactions on Components, Packaging, and Manufacturing Technology, Vol. 1, No. 2, pp. 154-162, Feb. 2011.

[4] A. Cataldo, E. Piuzzi, G. Cannazza, and E. De Benedetto, "Improvement and Metrological Validation of TDR Methods for the Estimation of Static Electrical Conductivity," IEEE Transactions on Instrumentation and Measurement, Vol. 59, No. 5, pp. 1207-1215, May 2010.

[5] Y. Wang, K. P. Cheung, R. Choi, and B.-H. Lee, "An Accurate Capacitance-Voltage Measurement Method for Highly Leaky Devices-Part II,” IEEE Transactions on Electron Devices, Vol. 55, No. 9, pp. 2437-2442, Sep. 2008.

[6] C.-H. Cheng, C.-H. Tsai, and T.-L. Wu, "A Novel Time Domain Method to Extract Equivalent Circuit Model of Patterned Ground Structures,” IEEE Microwave and Wireless Components Letters, Vol. 20, No. 9, pp. 486-488, Sep. 2010.

[7] J. A. Bishop, D. J. Pommerenke, and G. Chen, “A Rapid-Acquisition Electrical Time-Domain Reflectometer for Dynamic Structure Analysis,” IEEE Transactions on Instrumentation and Measurement, Vol. 60, No. 2, pp. 655-661, Feb. 2011.

[8] A. Cataldo, L. Catarinucci, L. Tarricone, F. Attivissimo, and E. Piuzzi, "A Combined TD-FD Method for Enhanced Reflectometry Measurements in Liquid Quality Monitoring,” IEEE Transactions on Instrumentation and Measurement, Vol. 58, No. 10, pp. 3534-3543, Oct. 2009.

[9] D. Kwon, M. H. Azarian, and M. Pecht, "Nondestructive Sensing of Interconnect Failure Mechanisms Using Time-Domain Reflectometry,” IEEE Sensors Journal, Vol. 11, No. 5, pp. 1236-1241, May 2011.

[10] M. K. Chen, C.-C. Tai, and Y.-J. Huang, "Nondestructive Analysis of Interconnection in Two-Die BGA Using TDR," IEEE transactions on instrumentation and measurement, Vol. 55, No. 2, pp. 400-405, Apr. 2006. 


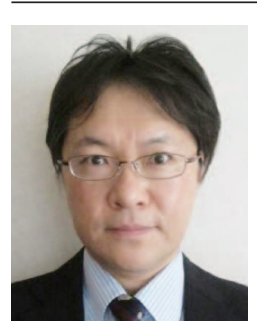

Keiji Matsumoto was born in Fukuoka, Japan. He received the B.E. Degree in electronic engineering and Doctor of Engineering from Fukuoka University. He is currently an associate professor in Department of Electrical and Electronic Engineering at Kitakyushu National College of Technology. His research interest is focused on the areas of high-frequency measurement and simulation, modeling, failure analysis of IC packaging, nondestructive testing.

\section{Hiroaki Otsuka}

Osamu Horiuchi

Young Gun Han

Woon Choi

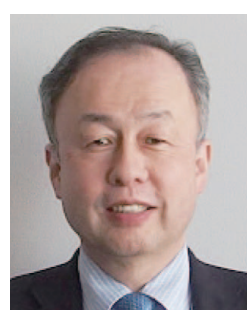

Hajime Tomokage was born in Yamaguchi, Japan in 1953. He received B.E. Degree in 1977, M.E. Degree in 1979, and Doctor of Engineering in 1982 from Kyushu University.

He joined Fukuoka University as a lecturer in 1982 and became associate professor in 1987. Since 1992, he has been a professor in Department of Electronics Engineering and Computer Science, Fukuoka University. His research area covers nanotechnology with carbon materials and high-frequency System-in-a-Package (SiP) design and evaluation.

Prof. Tomokage had been the president of the Japan Institute of Electronics Packaging from 2009 to 2011. He has been the chairman of the International Workshop on Microelectronics Assembling and Packaging (MAP) since 2001, and the director of Research Center for Three Dimensional Semiconductors since 2013. 